\title{
COMPARACIÓN DE LA ORDENACIÓN CONTINGENTE Y DEL EXPERIMENTO DE ELECCIÓN EN LA VALORACIÓN DE LAS FUNCIONES NO PRIVADAS DE LOS BOSQUES ${ }^{1}$
}

\author{
Joan Mogas Amorós, Pere Riera ${ }^{2}$
}

\begin{abstract}
RESUMEN: En este trabajo se presenta una comparación entre dos métodos de valoración de preferencias declaradas: la ordenación contingente y el experimento de elección. Se incluye una aplicación empírica en la que se valoran los impactos que una determinada forestación de terrenos agrícolas en Cataluña tendría sobre la función recreativa, de absorción de $\mathrm{CO}_{2}$ y de protección frente a la erosión de los bosques. Los resultados muestran diferencias entre los valores obtenidos a partir de los dos métodos, siendo los del método de elección significativamente mayores. Además, esta divergencia se mantiene cuando se comparan observaciones pairwise obtenidas directamente a través del método de elección con las simulaciones pairwise procedentes de la ordenación contingente.
\end{abstract}

Palabras Clave: valoración ambiental, ordenación contingente, experimento de elección, repoblación forestal

Códigos JEL: Q23, C90

\section{A COMPARISON OF THE CONTINGENT ORDERING AND CHOICE EXPERIMENT APPROACH FOR THE ASSESSMENT OF NON-PRIVATE FUNCTIONS OF THE FOREST.}

\footnotetext{
${ }^{1}$ Los autores agradecen la financiación recibida del Ministerio de Agricultura, Pesca y Alimentación (CICYT INIA FOA97-1646) y del proyecto MEDFOREX, y a los evaluadores anónimos por sus comentarios que han permitido mejorar este trabajo.

${ }^{2}$ Autor para correspondencia: Joan Mogas Amorós Departament d'Economia, Facultat de Ciències Econòmiques i empresarials Universitat Rovira i Virgili, Avgda. Universitat, 1. 43204 Reus e-mail: jma@fcee.urv.es Tfn. 977 759851. Fax. 977300661 Recibido en febrero de 2001. Aceptado en noviembre de 2001.
} 
ABSTRACT: This paper presents a comparison between two stated preference methods: the contingent ranking and the choice experiment. An empirical application is included, involving the valuation of the effects that would have a specific program to convert some agricultural land into forest land in Catalonia. The valuation exercise considers a forest recreational function, carbon storage, and erosion protection. The results show some differences between the values derived from the two methods, with the choice experiment values being significantly greater. Moreover, the differences are kept when comparing pairwise observations derived from the choice experiments with simulated pairwise observations derived from the contingent ranking.

Keywords: environmental valuation, contingent ranking, choice experiment, afforestation

\section{Introducción}

Los bosques son un bien particularmente complejo que generan un gran número de efectos externos. Además de la función productiva, básicamente de suministro de materias primas que afectan al ámbito privado, los bosques ofrecen toda una serie de funciones ambientales que afectan a la sociedad en su conjunto. Algunos ejemplos son la formación y conservación de los suelos, la absorción de $\mathrm{CO}_{2}$, la producción de oxígeno, la regulación del ciclo del agua, la creación de paisaje o la conservación de la biodiversidad. También ofrecen otras funciones que tienen demanda social, como las recreativas.

El principal problema al que a menudo se enfrenta el análisis económico de estas funciones no madereras es la ausencia de mercados directos donde observar su valoración por parte de los agentes. La economía ha desarrollado un conjunto de técnicas para estimar valores para los bienes sin mercado, siendo la valoración contingente el método más usado (Mitchell y Carson, 1989). Sin embargo, existen otros métodos de preferencias declaradas como la ordenación, la puntuación y la elección contingente, capaces de obtener preferencias sobre bienes ambientales y que, comparativamente, se han utilizado poco en la valoración medioambiental (véase como ejemplo de cada uno de ellos, Beggs et al., 1981, Roe et al., 1996 y Adamowicz et al., 1998). En lugar de ofrecer a las personas entrevistadas un único bien, en estos otros métodos se les ofrece múltiples bienes - o un único bien con diferentes niveles en sus 
atributos- y se les pregunta de una determinada manera para que expresen sus preferencias sobre el conjunto de bienes. Estos métodos presentan ventajas e inconvenientes. Para una discusión sobre ambas, véase, por ejemplo, Morrison et al. (1996).

En este trabajo se lleva a cabo una comparación entre dos métodos de preferencias declaradas: la ordenación contingente (contingent ranking) y el método de elección contingente entre parejas o experimento de elección (choice experiment). La aplicación empírica consiste en el cálculo de los valores asociados a cambios potenciales en las funciones recreativas, de absorción de $\mathrm{CO}_{2}$ y prevención de la erosión como resultado de un aumento de la superficie de bosques de Cataluña. Este trabajo difiere de anteriores aplicaciones de comparación entre métodos de preferencias (Mackenzie, 1993; Boxall et al.; 1996; Adamowicz et al., 1998; Hanley et al.,1998b) en que la ordenación contingente y el experimento de elección se realizan a partir de muestras separadas. El objetivo es comparar la diferencia en los valores de las disposiciones marginales a pagar que se obtiene usando estas dos técnicas por separado, respecto a los que se obtendrían si las observaciones pairwise se simularan a partir de las observaciones de ordenación contingente.

En la siguiente sección se presenta un breve repaso a la investigación previa en la aplicación del método de ordenación y elección contingente y en la comparación entre métodos de preferencias declaradas. En la sección tercera se desarrolla el modelo logit condicional como la base del análisis estadístico de los datos obtenidos a partir de la aplicación de los métodos de preferencias declaradas. Seguidamente se expone el diseño de los ejercicios de ordenación y elección y la forma de obtención de los datos. Los resultados obtenidos de la aplicación empírica de cada uno de los dos métodos se presentan por separado en la quinta sección. En la sexta, se comparan y se analizan las disposiciones marginales a pagar obtenidas a partir de los dos métodos. El último apartado incluye las conclusiones y las implicaciones de los resultados de la investigación. 


\section{Comparación entre experimento de elección y ordenación contingente}

Mientras en el experimento de elección se pide a la persona entrevistada que elija la opción preferida entre diferentes alternativas (véase, por ejemplo, Magat et al. 1988; Adamowicz et al., 1994; Boxall et al., 1996; Adamowicz et al., 1998; Hanley et al., 1998b o Rolfe et al., 2000), la ordenación contingente consiste en pedirle a la persona entrevistada que ordene un conjunto de alternativas según sus preferencias (véase, por ejemplo, Beggs et al., 1981; Desvouges et al., 1983; Lareau y Rae, 1989; Garrod y Willis, 1997; Garrod y Willis, 1998; o Mourauto y Foster, 1999). Las alternativas se componen de diferentes combinaciones de bienes (y/o sus atributos) y el coste asociado a cada una de ellas. Estos métodos presentan la ventaja respecto al método tradicional de valoración contingente de que permiten obtener el trade-off que los individuos hacen entre un conjunto amplio de atributos.

A pesar del número importante de estudios que han utilizado los métodos de preferencias declaradas, pocos llevan a cabo una comparación entre los mismos, por lo que los resultados disponibles son aún limitados para poder sacar algún tipo de evidencia respecto a la influencia de estos métodos en las valoraciones obtenidas. Además, en los estudios donde se comparan métodos directos, la tendencia es contrastar los resultados obtenidos con el método de valoración contingente con los procedentes de alguno de los otros métodos de preferencias declaradas. Ejemplos de trabajos que comparan los métodos de valoración contingente y ordenación contingente son Desvousges y Smith (1983), Riera (1995) y Mourato y Foster (1999), mientras que entre los trabajos donde los métodos comparados son el de elección y el de valoración contingente se encuentran los de Magat et al. (1988), Boxall et al. (1996), Adamowicz et al (1998), Hanley et al. (1998a) y Hanley et al. (1998b).

La comparación entre métodos de preferencias declaradas que no incluyan la valoración contingente es todavía muy escasa. Una de las primeras aplicaciones de comparación entre métodos de preferencias declaradas diferentes al de valoración contingente, es el trabajo de Mackenzie (1993). Compara la puntuación contingente (contingent rating), la ordenación contingente (contingent ranking) y dos modelos de elección contingente de parejas de alternativas (pairwise choice) para valorar las preferencias respecto a diferentes alternativas de viajes de caza. Sin embargo, para realizar esta comparación no 
lleva a cabo una aplicación empírica de cada uno de los métodos por separado, sino que establece unas reglas de decisión para usar los datos obtenidos mediante el método de puntuación para calcular el resto de los modelos. Al comparar los cuatro métodos y las medidas de disposición a pagar, encuentra que la puntuación contingente es el método que da lugar a estimaciones más eficientes y a intervalos de confianza más estrechos de las disposiciones marginales a pagar, siendo la ordenación contingente el segundo mejor. Encuentra, también, que el valor de las disposiciones marginales a pagar por los diferentes atributos valorados son similares entre los cuatro métodos que compara.

En cambio, en el trabajo que aquí se presenta, la estimación de las disposiciones marginales a pagar a través del método de ordenación y de elección se obtiene a partir de muestras separadas y representativas de la misma población. A diferencia del trabajo de Mackenzie (1993), los resultados muestran una divergencia significativa en las disposiciones a pagar cuando éstas se obtienen de la aplicación empírica de los dos métodos por separado en lugar de obtenerlas a partir de una misma aplicación.

\section{Especificación del modelo}

Tanto la estructura de la ordenación contingente como del experimento de elección entre parejas de alternativas, se pueden analizar utilizando el modelo de utilidad aleatoria. Si suponemos que la persona $i$ se enfrenta a $m$ alternativas mutua y exhaustivamente excluyentes, se puede representar la utilidad que la persona $i$ obtiene de la alternativa $j\left(U_{i j}\right)$ como:

$$
U_{i j}=V_{i j}+\varepsilon_{i j},
$$

donde $V_{i j}$ es la parte observable de la utilidad total (componente determinístico) que depende de las características de la alternativa y de la persona entrevistada. $\mathcal{E}_{i j}$ es la parte no observable (componente estocástico o aleatorio).

\subsection{Modelo para la elección de la alternativa preferida}

En el caso de que el individuo escoja la alternativa más preferida, elegirá la alternativa $j$ en lugar de otra alternativa $k$ si $U_{i j}>U_{i k}$ para toda $k \neq j$. Sin embargo, dado que las utilidades incluyen un componente estocástico, el investigador sólo puede describir la probabilidad de que la persona $i$ escoja la alternativa $j$ sobre las otras como: 


$$
\left.P_{i j}=\operatorname{Prob}\left\{V_{i j}+\varepsilon_{i j}>V_{i k}+\varepsilon_{i k} ; \quad \forall k \in C \backslash j\right\}\right\}
$$

donde $C$ representa el conjunto de todas las alternativas posibles y $C \backslash\{j\}$ denota el conjunto de todas las alternativas menos la alternativa $j$.

Bajo el supuesto de que los términos de error se distribuyen independiente e idénticamente con una distribución del tipo valor extremo (distribución Gumbel), se obtiene el denominado modelo logit condicional (McFadden,1973):

$$
P_{i j}=\frac{e^{\omega V_{i j}}}{\sum_{k=1}^{m} e^{\omega V_{i k}}}
$$

donde $\omega$ denota el parámetro escalar que es inversamente proporcional a la desviación estándar de las perturbaciones y que, generalmente, se normaliza a 1 al no ser identificable en la mayoría de aplicaciones (Ben-Akiva y Lerman, 1985).

\subsection{Modelo para la ordenación total o parcial}

Para estimar modelos de elección discreta a partir de datos obtenidos por ordenación contingente se tiene que identificar, en primer lugar, la relación correcta entre las probabilidades de elección y de ordenación. Si se cumple el axioma de Luce (Luce, 1959), los datos de la ordenación se pueden transformar en datos de elección. Es decir, la ordenación de $m$ alternativas es equivalente a una secuencia de $m-1$ problemas de elección independientes de la alternativa más preferida. Si las $\varepsilon_{i j}$ son independientes e idénticamente distribuidas con una distribución del tipo valor extremo (distribución Gumbel), y asumiendo el parámetro escalar igual a 1, la probabilidad de que el individuo $i$ realice la ordenación donde la alternativa 1 es preferida a la alternativa 2, la alternativa 2 es preferida a la 3 , y así sucesivamente, se obtiene a partir del producto de $m-1$ funciones de verosimilitud logit ordinarias:

$$
P_{i}(1,2, \ldots, m)=\prod_{j=1}^{m-1} \frac{e^{V_{i j}}}{\sum_{k=j}^{m} e^{V_{i k}}}
$$

A este modelo se le conoce como rank-ordered logit model o exploded logit model. 


\section{Diseño del ejercicio y obtención de los datos}

En nuestro estudio el objeto de estimación son las disposiciones a pagar por cambios en el bienestar debidos a variaciones en la calidad y/o la cantidad de algunos de los bienes públicos que los bosques proporcionan a la población. El estudio se centra en Cataluña, región que actualmente cuenta con una superficie arbolada de 1.332 .000 hectáreas, lo que representa aproximadamente un 40 por 100 de su superficie total. Tanto el experimento de elección como el de ordenación contingente se diseñan describiendo un cambio en la utilización de un $10 \%$ de la superficie de Cataluña, que pasaría de ser suelo agrícola a ser bosque mediante un programa de forestación o repoblación forestal, lo que implicaría tener bosques en una superficie equivalente a la mitad del territorio de Cataluña. Este programa implica algunos cambios que se suponen atractivos para las personas entrevistadas (disminución de la contaminación del aire y de la erosión) y otros que se suponen negativos (restricciones en algunas actividades recreativas y el precio a pagar). En la fase de confección de los cuestionarios se comprueba la preferencia del programa de forestación por parte de los encuestados. En concreto un 90 por ciento de las personas consultadas consideran la forestación planteada como buena o muy buena.

\subsection{Diseño de las alternativas de elección}

El primer paso para llevar a cabo los ejercicios de valoración es decidir los atributos o características que se utilizarán para construir las alternativas de forestación. A partir de la investigación previa en literatura forestal, consultas a investigadores en temas forestales, diferentes sesiones de trabajo (focus groups) y repetidas entrevistas previas a pequeñas muestras de la población, se determinaron los atributos y niveles asociados a algunos de los bienes públicos que los bosques producen a la población. Los atributos y niveles finales usados en ambos ejercicios aparecen descritos en el cuadro 1. 
Cuadro 1. Atributos y niveles utilizados en los ejercicios de preferencias declaradas

\begin{tabular}{|l|l|}
\hline ATRIBUTO & NIVELES \\
\hline PASEAR & \\
(Permitido) & Sí \\
\hline HACER PICNIC & Sí \\
(Permitido o no) & No \\
\hline CIRCULAR EN COCHE POR LOS CAMINOS & Sí \\
(permitido o no) & No \\
\hline BUSCAR SETAS & Sí \\
(Permitido o no) & No \\
\hline & 300.000 habitantes \\
GAS CO ELIMINADO AL AÑO $_{\text {Contaminación producida anualmente por una }}$ & 400.000 habitantes \\
ciudad de...) & 500.000 habitantes \\
\hline & 600.000 habitantes \\
DISMINUCIÓN DE LA EROSIÓN & Muy erosionadas (improductivas en 100 años) \\
( Los nuevos bosques en tierras ....) & Bastante erosionadas (improductivas en 300 años) \\
& Poco erosionadas (improductivas en 500 años) \\
& Muy poco erosionadas (improductivas en 700 años) \\
\hline & 1.000 pesetas \\
CONTRIBUCIÓN ANUAL & 2.000 pesetas \\
& 3.000 pesetas \\
& 4.000 pesetas \\
\hline
\end{tabular}

Si bien existen 7 atributos, el atributo PASEAR aparece en todas las alternativas de forestación ${ }^{3}$. Esto implica que, en realidad, a la hora de construir las alternativas, únicamente se emplean los restantes seis atributos. Así, si se trata cada atributo como discreto, existen $\left(2^{3} \times 4^{3}\right) \times\left(2^{3} \times 4^{3}\right)$ combinaciones posibles en el ejercicio de elección y $2^{3} \times 4^{3}$ opciones posibles en el ejercicio de ordenación contingente. En la práctica, el total de combinaciones, tanto en el ejercicio de elección (262.144) como en el de ordenación (512), es un número demasiado alto para poder esperar una evaluación coherente por parte de los encuestados.

En el ejercicio de elección, para reducir el número de alternativas a tamaños razonables, se utiliza un diseño factorial fraccionado de efectos principales más efectos interacción del universo de $\left(2^{3} \times 4^{3}\right) \times\left(2^{3} \times 4^{3}\right)$ combinaciones posibles (o alternativas de forestación). El diseño final consiste en 64 conjuntos de comparaciones pairwise. Sin embargo, puesto que no es realista suponer que la persona entrevistada pudiera escoger 64 veces entre combinaciones distintas de forestación, el diseño se divide en dieciséis versiones de 4 conjuntos de elección. Para ello se utilizan dos variables adicionales de 4 niveles tratadas como dos factores más en el diseño. Esta forma de agrupar asegura que cada

\footnotetext{
${ }^{3}$ Se optó por permitir mantener constante la opción de pasear en todas las alternativas de forestación, ya que en los focus group y en las encuestas pretest, se detectó que la opción de impedir pasear, caminar, por los bosques no era creíble. Probablemente esto es debido a que, si bien la mayoría de los bosques en Cataluña son de propiedad privada, actualmente el acceso al bosque es libre.
} 
versión de los conjuntos de elección es aproximadamente equivalente estadísticamente (Adamowicz et al., 1994). De esta manera a cada entrevistado se le presenta cuatro conjuntos de parejas de alternativas de forestación. Para cada conjunto de elección la persona entrevistada debe escoger entre no forestar (status quo) y las dos alternativas de forestación. Un ejemplo de uno de los conjuntos de elección presentados puede verse en el apéndice A.

En el método de ordenación, los escenarios de forestación se construyen a partir de un diseño ortogonal factorial fraccionado de efectos principales del total de $2^{3} \times 4^{3}$ combinaciones posibles (Hahn y Shapiro, 1966) que da lugar a 16 alternativas. En este caso también se considera que el número de alternativas a ordenar por cada entrevistado es demasiado grande, por lo que se divide en cuatro versiones de 4 alternativas añadiendo en el diseño una variable de cuatro niveles utilizada como un factor más. En cada versión se pide primero a la persona entrevistada que, de las cuatro alternativas presentadas, separe las alternativas de forestación con las que estaría de acuerdo de aquellas con las cuales estaría en desacuerdo. Una vez separadas, se le pide que ordene las alternativas con las que estaría de acuerdo de "más preferida" a "menos preferida". Análogamente, con las que estaría en desacuerdo de "menos mala" a "más mala". Un ejemplo de una de las alternativas de forestación se muestra en el apéndice B.

Debido a que el objetivo principal del trabajo es hacer una comparación de los resultados obtenidos a partir de la ordenación y la elección contingente, se diseña un cuestionario para cada método con tres partes diferenciadas. En la primera parte del cuestionario se presenta a la persona entrevistada los efectos que tendría la forestación y se le pregunta sobre su importancia relativa. En la tercera parte del cuestionario se incluyen varias preguntas acerca del uso del bosque y sobre características sociodemográficas de la persona entrevistada. La primera y la tercera parte del cuestionario son comunes a los dos métodos. La única diferencia se encuentra en la segunda parte de cuestionario, donde para una submuestra se aplica el experimento de elección y para la otra el método de ordenación contingente.

Por último, respecto al diseño, una cuestión relevante en nuestro trabajo es la consideración de si un bien ambiental como el bosque puede ser descrito en términos de los componentes individuales. Existen tres problemas al respecto. El primero es que el 
valor del bosque en su totalidad puede ser mayor o menor que la suma de los valores de sus atributos individualmente considerados (el valor del todo es mayor o menor que la suma de las partes). Esto ocurriría si los efectos interacción entre atributos fueran significativos. El segundo problema es la exclusión de atributos individuales de los bosques como puede ser el papel que juega el bosque en la regulación hídrica o en la conservación de la biodiversidad. Por último, hay que tener en cuenta que la forma en que el diseño experimental combina niveles de los atributos (diseño ortogonal factorial fraccionado) puede dar lugar a alternativas que no sean realistas ecológicamente. Respecto al primero de los problemas, el experimento de elección entre parejas se diseña de manera que permita calcular si determinados efectos interacción entre atributos son o no significativos. Por otra parte, tanto en el ejercicio de ordenación como en el de elección no se valora el bosque en su conjunto sino determinados atributos. En cuanto al último problema, tras realizar la selección de las alternativas, se comprueba que ninguna combinación era ecológicamente incompatible. En cambio, si se halla algún caso de una alternativa dominante sobre la otra en los conjuntos de elección (por ejemplo, cada atributo presenta mejores niveles menos en el pago requerido que es inferior). Existen básicamente dos estrategias estándar en la literatura para tratar la dominancia entre alternativas (Pearmin et al., 1991). Una consiste en mantener las alternativas dominantes a pesar de no aportar estas elecciones ninguna información acerca del trade-off entre los niveles de atributos, pero respetando la ortogonalidad. Esta estrategia permite a la vez identificar aquellos individuos que eligen las alternativas de forma aleatoria o ilógica. La otra estrategia consiste en eliminar aquellas opciones que son dominantes con la finalidad de situar a la persona entrevistada frente a un verdadero problema de elección, pero con una reducción en la ortogonalidad del diseño. En este trabajo se optó por la primer estrategia.

\subsection{Muestra y obtención de los datos}

La población objeto del estudio se limita a los habitantes de Cataluña mayores de edad afectados directamente por el aumento de la superficie de bosques (5.300.000 habitantes). La muestra de 2.000 individuos se selecciona de manera que sea representativa de esa población en términos del tamaño del municipio de residencia, edad y sexo de las personas entrevistadas. La muestra se divide a su vez en dos submuestras (de 1200 individuos para el experimento de elección y 800 para la 
ordenación) siguiendo ambas el mismo criterio de representatividad de la muestra general.

Las entrevistas son personales y realizadas a domicilio y se llevan a cabo la segunda mitad del año 1999. El porcentaje de respuesta en el experimento de elección es del 95 por ciento de la población entrevistada y del 78 por ciento en el de ordenación. Así, se obtienen 4.576 observaciones válidas para el experimento de elección. Ello corresponde a cuatro observaciones para cada individuo que responde a todas los conjuntos de elección y a menos observaciones para los que dejan algunos sin contestar. El número de observaciones válidas en el método de ordenación es de 626.

\subsection{Análisis estadístico}

El modelo de elección se estima utilizando el modelo logit condicional (McFadden, 1973) y el de ordenación contingente se estima utilizando el modelo rank-ordered logit (Beggs et al., 1981). En ambos modelos se supone que la función de utilidad $\mathrm{V}_{i j}$ es lineal en los parámetros y aditivamente separable. Se incluye como variables explicativas los atributos descritos en el cuadro 1 excepto PASEAR ya que no se puede identificar su efecto por separado del término constante.

Los atributos categóricos (picnic, circular en coche y recoger setas) se codifican como effects codes $(-1,1)$ en lugar de codificarlos como una variable ficticia clásica $(0,1)$ como es habitual codificarlos en los modelos de preferencias declaradas alternativos a la valoración contingente (Adamowicz et al., 1994). Los atributos cuantitativos se codifican de forma lineal y se centran en su media para reducir la colinealidad (Louviere y Woodworth, 1983).

Para poder comparar los resultados entre ambos métodos, en el modelo de ordenación se incluye también una alternativa de status quo a partir de la siguiente suposición: las alternativas con las cuales el individuo está de acuerdo son alternativas preferidas a la situación de status quo (no hacer la forestación), mientras que las alternativas con las que está en desacuerdo son menos preferidas que la situación de status quo. Esta hipótesis implica añadir una quinta alternativa. Tanto en el método de ordenación 
contingente como en el experimento de elección, los niveles de los atributos asociados a la alternativa de no forestación (status quo) se igualan a cero para todos los atributos.

\section{Resultados}

Los resultados del análisis logit de los datos obtenidos a partir del ejercicio de elección y de los obtenidos mediante la ordenación contingente se muestran en el cuadro 2. Los coeficientes estimados de los parámetros tienen signos consistentes con nuestras expectativas y excepto PICNIC el resto de variables tienen los mismos signos en ambos modelos. El signo positivo de las variable SETAS indica las preferencias positivas de la población para poder ir a buscar setas en los nuevos bosques, mientras que el acceso con vehículo a los bosques afecta negativamente la utilidad de los individuos. El signo positivo de $\mathrm{CO}_{2}$ indica una mejora de la utilidad a medida que aumenta la cantidad de $\mathrm{CO}_{2}$ absorbida por los nuevos bosques, mientras que el signo negativo de EROSION sugiere que la forestación en terrenos muy erosionados es preferida a la forestación en terrenos poco erosionados. El signo de la variable PRECIO es el esperado ya que la probabilidad de aceptar el pago de una contribución anual por un determinado tipo de forestación, disminuye a medida que éste aumenta. Por último, la constante tiene un valor positivo y significativo en los dos métodos. Este valor se puede interpretar como la mayor utilidad, manteniéndose todo el resto constante, asociada a un aumento de la superficie de bosques. Es decir, la población de Cataluña prefiere que se lleve a cabo la forestación que quedarse con la superficie actual.

\section{Cuadro 2. Coeficientes de los modelos de elección y de ordenación contingente}

\begin{tabular}{|c|c|c|}
\hline Variable (Atributo) & Modelo de elección & Modelo de Ordenación \\
\hline CONSTANTE & $\begin{array}{c}1,044066565^{*} \\
(8,860)\end{array}$ & $\begin{array}{c}1,353152419^{*} \\
(47,965)\end{array}$ \\
\hline PICNIC & $\begin{array}{c}0,040435447 * * \\
(1,920)\end{array}$ & $\begin{array}{c}-0,000020720 \\
(-0,001)\end{array}$ \\
\hline COCHE & $\begin{array}{c}-0,080171129 * \\
(-3,799)\end{array}$ & $\begin{array}{c}-0,067747220 * \\
(-2,473)\end{array}$ \\
\hline SETAS & $\begin{array}{c}0,053615529 * \\
(2,560)\end{array}$ & $\begin{array}{c}0,023214428 \\
(0,826)\end{array}$ \\
\hline $\mathrm{CO}_{2}$ & $\begin{array}{c}0,000000775^{*} \\
(4,020)\end{array}$ & $\begin{array}{c}0,000000142 \\
(0,825)\end{array}$ \\
\hline EROSION & $\begin{array}{c}-0,000301284 * \\
(-3,179)\end{array}$ & $\begin{array}{c}-0,000400287 * \\
(-3,283)\end{array}$ \\
\hline PRECIO & $\begin{array}{c}-0,000111645^{*} \\
(-5,835)\end{array}$ & $\begin{array}{c}-0,000204663^{*} \\
(-8,815)\end{array}$ \\
\hline Verosimilitud Log & $-4600,954$ & $-2663,689$ \\
\hline
\end{tabular}




$\begin{array}{lcc}\text { Veros.Log. Rest. } & -4634,774 & -2692,752 \\ \text { ICV } & 0,00729 & 0,01079 \\ \text { VZ } & 0,02175 & 0,02320 \\ \chi^{2} & 67,64 & 58,12 \\ \text { Observaciones } & 4.576 & 626\end{array}$

Valores del estadístico $t$ entre paréntesis

*,**Significativo para un nivel de error máximo del $5 \%$ y $10 \%$ respectivamente

ICV: Índice de Cociente de Verosimilitudes o Pseudo-R ${ }^{2}$ de McFadden (1973)

VZ: Pseudo-R² de Veall y Zimmermann (1992)

Si se compara los estadísticos $t$ de los coeficientes en los dos modelos, el de elección presenta un mayor número de variables significativas ya que únicamente PICNIC no es significativa, mientras que en el modelo de ordenación contingente, además de PICNIC y al mismo nivel de confianza, no son significativas las variables SETAS y $\mathrm{CO}_{2}$. Sin embargo, cuando se realiza esta comparación, hay que tener en cuenta que el número de observaciones en el experimento de elección es mucho mayor que el de la ordenación.

Ambos modelos presentan una bondad de ajuste relativamente reducida de acuerdo con Índice de Cocientes de Verosimilitudes, si bien es algo mayor cuando utilizamos otros Pseudo- $\mathrm{R}^{2}$ como el de Veall y Zimmermann (1992) ${ }^{4}$. El estadístico del cociente de verosimilitudes rechaza a menos de un 5 por ciento de significación la hipótesis de que todas las pendientes del modelo son cero ${ }^{5}$. El relativo bajo Pseudo- $\mathrm{R}^{2}$ puede explicarse en gran parte debido a la presencia de una elevada heterogeneidad en las preferencias de los individuos.

\section{Comparación de los modelos}

\subsection{Contraste de hipótesis sobre la equivalencia entre métodos}

Al ser el comportamiento de los individuos que implica la teoría de la utilidad aleatoria el mismo en los dos métodos, éstos deberían proporcionar medidas de bienestar similares. En este apartado se contrasta si la disposición marginal a pagar (DAP marginal) por cada atributo es significativamente distinta en el método de elección que en el método de ordenación contingente.

\footnotetext{
${ }^{4}$ Valores del Pseudo- $\mathrm{R}^{2}$ entre 0,2 y 0,4 se consideran indicativos de un ajuste del modelo muy alto. Simulaciones de Domencich y McFadden (1975) muestran una equivalencia de este rango a un intervalo de entre 0,7 y 0,9 de una función lineal.

${ }^{5}$ El valor crítico de una chi-cuadrado con 6 grados de libertad es de 18,55 para un nivel de significación del 5 por 100.
} 
El valor marginal de un atributo es la disposición a pagar por un cambio unitario en este atributo manteniéndose el resto constante. Si la función de utilidad $\mathrm{V}_{j}$ tiene la siguiente forma lineal:



la DAP marginal se calcula como el ratio del parámetro estimado dividido por el negativo del precio. Por ejemplo, manteniendo la utilidad constante, la DAP marginal para una reducción de $\mathrm{CO}_{2}$ equivalente a las emisiones que, en promedio, un ciudadano español añade al aire cada año, es el cociente $-\beta_{C O 2} / \beta_{P R E C I O}$.

De esta manera la hipótesis nula y alternativa es nuestro caso son:

$\mathrm{H}_{\mathrm{o}}: \beta(\mathrm{C})_{\mathrm{i}} / \beta(\mathrm{C})_{\mathrm{PRECIO}}=\beta(\mathrm{R})_{\mathrm{i}} / \beta(\mathrm{R})_{\mathrm{PRECIO}}$

$\mathrm{H}_{\mathrm{A}}: \beta(\mathrm{C})_{\mathrm{i}} / \beta(\mathrm{C})_{\mathrm{PRECIO}} \neq \beta(\mathrm{R})_{\mathrm{i}} / \beta(\mathrm{R})_{\mathrm{PRECIO}}$

donde $\beta_{\mathrm{i}}$ es el parámetro estimado de un atributo no monetario y $\beta_{\mathrm{PRECIO}}$ es el parámetro estimado del atributo PRECIO. C representa el modelo de elección y R representa el modelo de ordenación contingente.

En el cuadro 3 se muestran las DAP marginales y los intervalos de confianza de los diferentes atributos no monetarios para el modelo condicional logit del ejercicio de elección y de ordenación contingente. Debido a que las estimaciones de las disposiciones a pagar son funciones no lineales del vector de parámetros estimados, no se puede utilizar el procedimiento normal para la estimación de los intervalos de confianza por lo que es necesario recurrir a procedimientos alternativos para obtenerlos. En este trabajo se utiliza el procedimiento desarrollado por Krinsky y Robb (1986) para calcular intervalos de confianza. Este procedimiento simula la distribución de probabilidad a partir de repetidas extracciones aleatorias de la distribución normal multivariante parametrizada a partir de las estimaciones de los coeficientes y la matriz de covarianzas de los modelos estimados en la sección anterior. En este caso se realizan 1000 extracciones aleatorias con las que se calculan los cocientes PICNIC/PRECIO, SETAS/PRECIO, COCHE/PRECIO, PASEAR/PRECIO, $\mathrm{CO}_{2} / \mathrm{PRECIO}$, EROSION/PRECIO. Las distribuciones empíricas generadas a partir de dichas 
extracciones se emplean posteriormente para definir los intervalos de confianza de las DAP marginales siguiendo la aproximación de percentiles de Efron y Tibshirani (1993).

Cuadro 3. Disposiciones marginales a pagar (en ptas. de 1999): elección contingente (DAP marginal ${ }_{C}$ ) y ordenación contingente (DAP marginal $_{\mathbf{R}}$ ).

\begin{tabular}{|c|c|c|c|}
\hline Variables & $D^{D A P}$ marginal $_{C}$ & $D A P$ marginal $_{R}$ & $\frac{D A P \text { marginal }_{C}}{D A P \text { marginal }_{R}}$ \\
\hline PICNIC & $\begin{array}{c}724,360^{\#} \\
(-18,0471561,060)\end{array}$ & $\begin{array}{c}-0,202^{\# \#} \\
(-547,587486,394)\end{array}$ & 3.586 \\
\hline COCHE & $\begin{array}{c}-1436,166 \\
(-2587,092-652,805)\end{array}$ & $\begin{array}{c}-662,036 \\
(-1.310,438-139,600)\end{array}$ & 2,15 \\
\hline SETAS & $\begin{array}{c}960,454 \\
(216,7481891,756)\end{array}$ & $\begin{array}{c}226,850^{\# \#} \\
(-321,896752,656)\end{array}$ & 4,25 \\
\hline $\mathrm{CO}_{2}$ & $\begin{array}{c}0,0069 \\
(0,0030,011)\end{array}$ & $\begin{array}{c}0,0006^{\# \#} \\
(-0,0010,002)\end{array}$ & 10 \\
\hline EROSION & $\begin{array}{c}-2,698 \\
(-5,240-1,038)\end{array}$ & $\begin{array}{c}-1,955 \\
(-3,561-0,772)\end{array}$ & 1,38 \\
\hline
\end{tabular}

\footnotetext{
\#Coeficientes no significativos de esta variable en el modelo de elección contingente

\#\#Coeficientes no significativos de estas variables en el modelo de ordenación contingente Intervalos de confianza al 95 por ciento entre paréntesis

Nota: los valores de las DAP marginales de los atributos PICNIC, COCHE y SETAS, corresponden a los cálculos de la DAP marginal utilizando la transformación de effects codes utilizada para obtener los estimadores de estos atributos tanto en el experimento de elección como de ordenación.
}

La DAP marginal se calcula respecto a las pesetas por año que de forma indefinida estaría dispuesta a pagar la persona entrevistada. Valores marginales positivos (negativos) de un atributo indican que la persona estaría mejor con incrementos (disminuciones) en los niveles de estos atributos. Los valores de PICNIC, SETAS y COCHE corresponden a un cambio discreto de poder a no poder hacer picnic, recoger setas o circular en coche en los nuevos bosques. Para el atributo $\mathrm{CO}_{2}$, es la disposición a pagar por una reducción de este gas equivalente a las emisiones de $\mathrm{CO}_{2}$ que en promedio un ciudadano español añade al aire cada $a \tilde{n}{ }^{6}$. Por último el valor de EROSION se interpreta como la pérdida de la productividad del suelo en un año.

Los resultados del cuadro 3 muestran que existe una variación considerable entre las disposiciones marginales a pagar obtenidas mediante cada uno de los dos métodos.

\footnotetext{
${ }^{6}$ Los cálculos se hicieron en base al total de emisiones de $\mathrm{CO}_{2}$ a la atmosfera en Cataluña el 1995. Según datos proporcionados por el Departament de Medi Ambient de la Generalitat de Cataluña (1996) éstas fueron de 38.799.378 toneladas, lo que equivale a unas emisiones por cápita de unas 6,8 toneladas anuales.
} 
Aunque la utilidad asociada a cada atributo tiene el mismo signo (con la excepción de PICNIC), todas las estimaciones obtenidas a partir del método de elección son mayores en valores absolutos que las del método de ordenación. Así, por ejemplo, para el atributo $\mathrm{CO}_{2}$, la DAP marginal es casi 10 veces mayor en el ejercicio de elección que en el de ordenación.

Para contrastar la hipótesis de equivalencia entre las medias de las disposiciones marginales a pagar, se utiliza el contraste no paramétrico propuesto por Poe et al. (1997). Consiste en calcular el intervalo de confianza de la diferencia entre las dos variables aleatorias de interés (DAP marginal $_{R}$ y DAP marginal ${ }_{C}$ ) y reformular la hipótesis nula de igualdad entre las dos variables como la hipótesis nula que la diferencia entre ellas es igual a cero. A continuación se puede utilizar una aproximación bootstrapping para contrastar la hipótesis nula.

El cuadro 4 muestra los resultados del contraste de hipótesis para las medidas de bienestar consideradas. Para todos los casos la equivalencia entre las disposiciones marginales a pagar obtenidas a partir de la ordenación y elección contingente se rechaza desde el punto de vista estadístico. Los intervalos de confianza al 95 por ciento indican que las diferencias son grandes, lo cual se interpreta como que el método de elección entre parejas da lugar a una valoración significativamente mayor de los atributos examinados.

Cuadro 4. Equivalencia entre métodos en la valoración de todos los atributos $\mathrm{H}_{0}$ : DAP marginal $_{\mathrm{C}}=$ DAP marginal $_{\mathrm{R}} ; \mathrm{H}_{\mathrm{A}}$ : DAP marginal $_{\mathrm{C}}>$ DAP marginal $_{\mathrm{R}}$

$\begin{array}{ccc}\begin{array}{c}\text { DAP marginal } \\ \text { menos }\end{array} & \begin{array}{c}95 \% \\ \text { InP } \text { marginal }_{R}\end{array} & \begin{array}{c}\text { Nivel de confianza } \\ \text { aproximado }\end{array} \\ \text { PICNIC } & 471,9701178,358 & 0,000 \\ \text { COCHE } & -1.389,978-427,270 & 0,000 \\ \text { SETAS } & 463,4101284,744 & 0,000 \\ \mathrm{CO}_{2} & 0,0040,009 & 0,000 \\ \mathrm{EROSION} & -1,725-0,2745 & 0,000\end{array}$

Al comparar los coeficientes de los distintos modelos hay que tener en cuenta el supuesto utilizado de igualar a 1 el parámetro escalar $\omega$ de la ecuación (3.1) en los dos 
modelos econométricos, como se supone normalmente en la práctica. Sin embargo, el factor escalar en los modelos logit condicionales está inversamente relacionado con la varianza de las influencias no observadas (recogidas en los términos de error) lo que significa que modelos con un ajuste mejor tienen factores escalares mayores. Ello supone que, si bien la comparación se hace bajo la hipótesis de $\omega$ igual a 1, la correcta comparación entre los coeficientes de los dos modelos implicaría contrastar antes esta hipótesis, aislando así la posible influencia de $\omega$ en las diferencias observadas (Swait y Louviere, 1993).

\subsection{Diferencias en las medidas de bienestar debidas al diseño estadístico}

Si bien en el anterior apartado se comprueba una diferencia significativa entre las DAP marginales obtenidas mediante el ejercicio de elección y de ordenación, el distinto diseño experimental utilizado en cada uno de ellos no permite asegurar que estas diferencias sean debidas únicamente al método de valoración. Para ello hubiera sido necesario utilizar el mismo diseño factorial fraccionado. Por ejemplo, utilizar tanto en la aplicación del método de elección como en el de ordenación el diseño factorial fraccionado de efectos principales ${ }^{7}$.

El diseño factorial fraccionado de efectos principales para el ejercicio de elección consiste en 16 conjuntos de comparaciones pairwise del universo de $\left(2^{3} \times 4^{3}\right) \times\left(2^{3} \times 4^{3}\right)$ combinaciones posibles. Debido a que las 16 comparaciones de parejas necesarias no están incluidas dentro de las 64 combinaciones realmente utilizadas para poder calcular los efectos principales y las interacciones, no se puede inferir el diseño de efectos principales del diseño utilizado en el método de elección. Por ello, y como aproximación, se simula este diseño mediante la técnica del bootstrap ${ }^{8}$ (Efron y Tibshirani, 1993). La simulación consiste en 1000 extracciones aleatorias de 16 conjuntos del total de 64 con las que se calculan el vector de los coeficientes y la matriz de covarianzas de los atributos del modelo de elección simulado de efectos principales.

\footnotetext{
${ }^{7}$ La razón de haber utilizado para el experimento de elección un diseño con un número mayor de alternativas que las que corresponderían a un diseño de efectos principales, responde a un programa de investigación que va más allá del objetivo de este artículo.

${ }^{8}$ Los autores agradecen la ayuda recibida del profesor Jordan Louviere y del profesor Victor Adamowicz en la aproximación del diseño factorial de efectos principales para el modelo de elección.
} 
La hipótesis nula a contrastar es si las DAP marginales que se obtienen del modelo de efectos principales en el método de ordenación (DAP marginal $_{R}$ )son iguales a las DAP marginales obtenidas a partir del modelo simulado de efectos principales en el método de elección (DAP marginal CS $_{\text {) }}$. La aceptación de esta hipótesis parecería sugerir que la diferencia significativa obtenida en el apartado anterior entre las DAP marginales es debida al diseño utilizado, ya que cuando éste fuera el mismo las DAP no diferirían según el método de valoración. Para comprobar la hipótesis de equivalencia se utiliza el contraste no paramétrico propuesto por Poe et al. (1997) explicado previamente. El cuadro 5 muestra los resultados de este contraste de hipótesis para cada uno de los atributos relacionados con la forestación.

Cuadro 5. Equivalencia entre diseños en la valoración de todos los atributos $\mathrm{H}_{\mathbf{0}}$ : DAP marginal $_{\mathrm{CS}}=$ DAP marginal $_{\mathrm{R}} ; \mathrm{H}_{\mathrm{A}}:$ DAP marginal $_{\mathrm{CS}}>$ DAP $_{\text {marginal }} \mathrm{R}_{\mathrm{R}}$

\begin{tabular}{ccc}
$\begin{array}{c}\text { DAP marginal } \\
\text { menos } \\
\text { DAP marginal }\end{array}$ & $\begin{array}{c}95 \% \\
\text { Intervalo de confianza }\end{array}$ & $\begin{array}{c}\text { Nivel de confianza } \\
\text { aproximado }\end{array}$ \\
\hline PICNIC & $2921.460,208$ & 0,003 \\
COCHE & $-1.642,509-253,190$ & 0,005 \\
SETAS & $-10,4021.322,221$ & 0,027 \\
$\mathrm{CO}_{2}$ & $0,0030,009$ & 0,000 \\
$\mathrm{EROSION}$ & $-1,7041,262$ & 0,288
\end{tabular}

Como se observa, excepto para EROSION, se rechaza significativamente la equivalencia entre la DAP marginal $\mathrm{C}_{\mathrm{CS}}$ y la DAP marginal $_{\mathrm{R}}$. Este resultado sugiere que la explicación principal de la diferencia entre las disposiciones a pagar que se obtienen entre métodos a partir de los datos originales es el método de preferencias declaradas utilizado, y no el diferente diseño, ya que cuando se comparan las DAP marginales utilizando un diseño factorial fraccionado de efectos principales las diferencias se mantienen. Sin embargo, no hay que olvidar que esta comparación se basa en una aproximación, ya que el diseño correspondiente al experimento de elección es simulado, por lo que esta sugerencia debe tomarse con precaución y confirmarse con un experimento de elección ad hoc con un diseño de efectos principales.

6.3. Diferencias entre las medidas de bienestar obtenidas a partir del mismo método de preferencias declaradas 
En el ya mencionado trabajo de Mackenzie (1993), a partir de la comparación de los estadísticos $t$ de los coeficientes de los atributos en los cuatro modelos que considera, encuentra que la puntuación contingente es el método más eficiente en el sentido de que los errores estándar de los estimadores son menores. La mayor eficiencia comporta que las medidas sobre la DAP marginal obtenidas a partir de la puntuación tengan intervalos de confianza más pequeños. En esta comparación de los estadísticos $t$, la ordenación contingente domina sobre los dos modelos de comparación de parejas. El supuesto fundamental en el que se sustentan estos resultados es que los modelos de preferencias declaradas por separado dan lugar a las mismas preferencias que si los datos se obtuvieran del mismo modelo y que las únicas diferencias entre ellos y las medidas de las DAP, proceden de la forma en que se utilizan los datos para representar las preferencias.

En este apartado se examina esta hipótesis con los datos obtenidos de la aplicación empírica del método de ordenación y elección asociada a la forestación de la superficie de Cataluña. En concreto, las hipótesis nula y alternativa son respectivamente:

$\mathrm{H}_{\mathrm{o}}: \beta(\mathrm{C})_{\mathrm{i}} / \beta(\mathrm{C})_{\mathrm{PRECIO}}=\beta(\mathrm{CR})_{\mathrm{i}} / \beta(\mathrm{CR})_{\mathrm{PRECIO}}$

$\mathrm{H}_{\mathrm{A}}: \beta(\mathrm{C})_{\mathrm{i}} / \beta(\mathrm{C})_{\mathrm{PRECIO}} \neq \beta(\mathrm{CR})_{\mathrm{i}} / \beta(\mathrm{CR})_{\mathrm{PRECIO}}$

donde $\beta_{\mathrm{i}}$ es el coeficiente de un atributo no monetario y $\beta_{\text {PRECIO }}$ es coeficiente de la variable PRECIO, C denota el modelo de elección y CR representa el modelo de elección simulado a partir del modelo de ordenación contingente.

Para poder realizar este contraste se construye en primer lugar una serie simulada de elecciones entre parejas de alternativas a partir de las respuestas del método de ordenación contingente. Esta aproximación resulta plausible dada la utilización de los mismos atributos y niveles en los dos métodos empleados. La idea es comparar las alternativas de dos en dos dentro de cada ordenación de alternativas, teniendo en cuenta la ordenación relativa de cada par y si la persona entrevistada estaría de acuerdo o no con una determinada alternativa. Así, por ejemplo, supongamos que en la ordenación de 4 alternativas el individuo ha respondido que estaría de acuerdo con las alternativas 1 y 2; en desacuerdo con la 3 y la 4; y que, a la vez, prefiere la 1 a la 2 y la 3 a la 4 . A partir de esta ordenación se puede construir la secuencia de 6 pares de alternativas, incluyendo la opción de status quo, que se muestra en el cuadro 6. 


\section{Cuadro 6. Ejemplo de simulación de elecciones entre parejas} a partir de una serie ordenada de alternativas

\begin{tabular}{|c|c|c|c|}
\hline $\begin{array}{c}\text { ALTERNATIVA } \\
\text { A }\end{array}$ & $\begin{array}{c}\text { ALTERNATIVA } \\
\text { B }\end{array}$ & $\begin{array}{c}\text { STATUS } \\
\text { QUO }\end{array}$ & $\begin{array}{c}\text { OPCIÓN } \\
\text { ELEGIDA }\end{array}$ \\
\hline 1 & 2 & SQ & 1 \\
\hline 1 & 3 & SQ & 1 \\
\hline 1 & 4 & SQ & 1 \\
\hline 2 & 3 & SQ & 2 \\
\hline 2 & 4 & SQ & 2 \\
\hline 3 & 4 & SQ & SQ \\
\hline
\end{tabular}

De esta manera es posible comparar la divergencia entre los resultados obtenidos de los datos originales del modelo de elección entre parejas y los simulados a partir de la aplicación de otro método (en este caso el método de ordenación contingente). Cuando se relacionan modelos de elección discreta con los datos de ordenación, se supone que el comportamiento de elección de cada ordenación cumple el axioma de elección de Luce (Luce, 1959), por lo que la probabilidad de una ordenación puede fácilmente relacionarse con las probabilidades de elección y la estructura condicional logit da lugar a un modelo apropiado para la estimación de preferencias (Beggs et al., 1981; Chapman y Staelin, 1982). Sin embargo, el supuesto que hay detrás tanto del modelo logit condicional y del modelo rank-ordered logit de que los términos de error de la función de utilidad del individuo se distribuyen independiente e idénticamente con una distribución del tipo valor extremo (distribución Gumbel), implica la hipótesis de independencia de las alternativas irrelevantes. Esta propiedad, si bien simplifica el proceso de estimación, implica homogeneidad en las preferencias de la población, por lo que sería deseable una mayor investigación a este respecto.

En primer lugar, y antes de contrastar la anterior hipótesis, se lleva a cabo la estimación logit condicional de la serie simulada de elecciones entre parejas. En el cuadro 7 se resumen los principales resultados de esta estimación.

Si se comparan estos resultados con los presentados en el cuadro 2, se observa que los parámetros estimados en el modelo de elección a partir de los datos de ordenación tienen el mismo signo que el modelo de elección original y conjuntamente son significativamente diferentes de cero. PICNIC, SETAS y $\mathrm{CO}_{2}$ no son estadísticamente significativos.

En términos de los valores de los estadísticos $t$, el modelo de elección simulado a partir de los datos de ordenación es más eficiente que el modelo de ordenación. Este resultado 
contradice los resultados de Mackenzie (1993) donde a partir de los datos obtenidos de la aplicación del método de puntuación muestra la mayor eficiencia relativa en la estimación del modelo de ordenación respecto las comparaciones pairwise.

\section{Cuadro 7. Coeficientes del modelo de elección a partir de los datos de ordenación contingente}

\begin{tabular}{lc} 
Variable (Atributo) & Modelo de elección \\
\hline CONSTANTE & $1,085342402^{*}$ \\
& $(8,677)$ \\
PICNIC & 0,030806217 \\
COCHE & $(1,353)$ \\
SETAS & $-0,093458203^{*}$ \\
CO & $(-4,080)$ \\
EROSION & 0,020968066 \\
PRECIO & $(0,911)$ \\
Verosimilitud Log & 0,000000269 \\
Veros.Log. Rest. & $(1,313)$ \\
ICV & $-0,000320088^{*}$ \\
VZ & $(-3,112)$ \\
$\chi{ }^{2}$ & $-0,000228508^{*}$ \\
Observaciones & $(-11,013)$ \\
\hline Valos & $-3626,050$ \\
& $-3659,516$ \\
& 0,00914 \\
& 0,02342
\end{tabular}

Valores del estadístico $t$ entre paréntesis

*Significativo para un nivel de error máximo del $5 \%$

ICV: Índice de Cociente de Verosimilitudes o Pseudo- ${ }^{2}$ de McFadden (1973)

VZ: Pseudo-R ${ }^{2}$ de Veall y Zimmermann (1992)

Por otra parte las estimaciones de las disposiciones a pagar por los diferentes atributos y sus respectivos intervalos de confianza del modelo de elección simulado a partir de los datos de ordenación, calculados con 1.000 repeticiones siguiendo el procedimiento de Krinsky y Robb (1986), se muestran en el cuadro 8.

Como se puede apreciar, si comparamos estas DAP marginales con las que se obtienen en el cuadro 3, de nuevo se da una variación importante entre las disposiciones marginales a pagar obtenidas a través de los distintos métodos y para todos los atributos 
no monetarios. En valores absolutos, las DAP marginales del modelo de elección original (DAP marginal ${ }_{C}$ ) siguen siendo mayores que las de los otros dos modelos, mientras que las derivadas a partir de los datos de ordenación (DAP marginal ${ }_{R C}$ ) están más cerca de las derivadas del modelo de ordenación (DAP marginal ${ }_{\mathrm{R}}$ ).

$\begin{aligned} & \text { Cuadro 8. Disposiciones marginales a pagar } \\ & \text { (en ptas. de 1999) para el modelo de elección }\end{aligned}$
contingente a partir de los datos del método de
ordenación contingente (DAP marginal
RC)

\footnotetext{
\#\#\# Coeficientes no significativos de esta variable en el modelo de elección a partir del modelo de ordenación Intervalos de confianza al 95 por ciento entre paréntesis Nota: los valores de las DAP marginal de los atributos PICNIC, COCHE y SETAS, corresponden a los cálculos de la DAP marginal utilizando la transformación de effects codes utilizada para obtener los estimadores de estos atributos tanto en el experimento de elección como de ordenación.
}

Para comprobar si estas diferencias entre las medidas de bienestar obtenidas del modelo

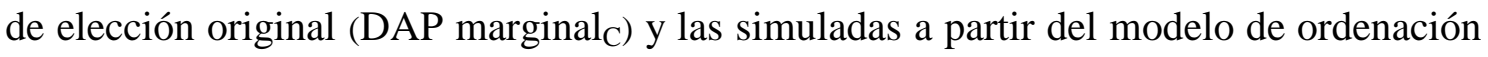
$\left(\right.$ DAP $_{\text {marginal }}{ }_{\mathrm{RC}}$ ) son significativas, se reformula la hipótesis nula de igualdad entre las disposiciones marginales a pagar como la hipótesis nula de que la diferencia entre ellas es igual a cero (Poe et al., 1997). El cuadro 9 muestra los resultados de este contraste de hipótesis para cada uno de los atributos relacionados con la forestación. También aquí se rechaza la equivalencia entre la DAP marginal $_{C}$ y la DAP marginal ${ }_{R C}$. 
Cuadro 9. Equivalencia entre métodos en la valoración de todos los atributos

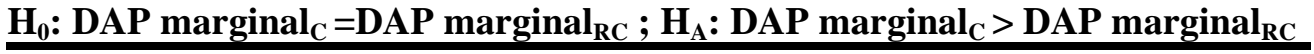

\begin{tabular}{ccc}
\hline $\begin{array}{c}\text { DAP marginal } \\
\text { menos } \\
\text { DAP marginal }\end{array}$ & $\begin{array}{c}95 \% \\
\text { Intervalo de confianza }\end{array}$ & $\begin{array}{c}\text { Nivel de confianza } \\
\text { aproximado }\end{array}$ \\
\hline PICNIC & $70,693969,074$ & 0,011 \\
SETAS & $377,2201447,925$ & 0,001 \\
COCHE & $-1.480,760-144,702$ & 0,000 \\
$\mathrm{CO}_{2}$ & $0,0020,010$ & 0,000 \\
EROSION & $-2,892-0,392$ & 0,000 \\
\hline
\end{tabular}

En definitiva, las disposiciones marginales a pagar por los diferentes atributos relacionados con un aumento de la superficie de bosques de Cataluña obtenidas con el método de elección entre pares de alternativas son significativamente mayores que las estimadas a partir del método de ordenación contingente. Este resultado se mantiene cuando se compara las DAP marginales estimadas a partir de la aplicación del método de elección con las DAP marginales simuladas a partir del método de ordenación. Al igual que el trabajo de Mackenzie (1993), al comparar las DAP marginales del método de elección y de ordenación estimadas a partir de los mismos datos se comprueba que los valores son similares. Sin embargo, las diferencias que se obtienen entre estas disposiciones cuando los datos proceden de muestras y ejercicios separados son significativas. Ello cuestiona el supuesto de este autor de que los métodos de preferencias declaradas utilizados en aplicaciones diferentes dan lugar a las mismas preferencias que si los datos se obtuvieran a partir de un solo método y se extrapolaran. Sin embargo, tanto los resultados de este estudio como los de Mackenzie deben tomarse con cautela, ya que el número de observaciones utilizadas en cada método es diferente, y esto influye en el valor de los errores estándar y por tanto en la significatividad de los estimadores. 


\section{Conclusiones}

A pesar de la creciente popularidad de los métodos de preferencias declaradas, la literatura en torno a los métodos de valoración de bienes de no mercado ofrece pocas comparaciones de las medidas de bienestar obtenidas. Este trabajo aporta un contraste de la equivalencia entre dos métodos de valoración de preferencias declaradas, el experimento de elección y la ordenación contingente. Se estiman las disposiciones marginales a pagar por diferentes atributos asociados a un aumento hipotético de la superficie de bosques de Cataluña, administrando cada uno de los métodos a una muestra separada de la población.

Los resultados muestran que aunque los dos métodos de preferencia declarada analizados en este trabajo comparten la misma base teórica en el modelo de utilidad aleatoria, la elección del método da lugar a diferencias significativas en las estimaciones de bienestar obtenidas. Este resultado está en la misma línea que la mayoría de estudios publicados sobre comparaciones entre otros métodos de preferencias declaradas. En concreto para cada uno de los métodos y los atributos forestales que se valoran en este trabajo se obtienen las DAP marginales que se muestran en el cuadro 9. Las DAP marginales se calculan respecto a las pesetas o euros que anualmente y de forma indefinida estaría dispuesto a pagar el ciudadano medio de Cataluña. Los valores de PICNIC, SETAS y COCHE corresponden a un cambio discreto de poder a no poder hacer picnic, recoger setas o circular en coche en los nuevos bosques; para el $\mathrm{CO}_{2}$, es la disposición a pagar por una reducción de este gas equivalente a las emisiones de $\mathrm{CO}_{2}$ que en promedio añade al aire cada año una ciudad de 100.000 habitantes $^{9}$; finalmente, EROSION se interpreta como el valor de la pérdida de productividad del suelo en un año, o bien, en signo positivo, como la disposición a pagar para que el suelo sea productivo un año más.

\footnotetext{
${ }^{9}$ Suponiendo una relación lineal entre la reducción anual de $\mathrm{CO}_{2}$ y la disposición a pagar por ésta.
} 
Cuadro10. Disposiciones marginales a pagar del experimento de elección (DAP marginal $\left._{C}\right)$ y de la ordenación contingente (DAP marginal $\left._{\mathbf{R}}\right)$.

\begin{tabular}{lcccc}
\hline Variables & $\begin{array}{c}\text { DAP marginal } \\
\text { (ptas. de 1999) }\end{array}$ & $\begin{array}{c}\text { DAP marginal } \\
\text { (Euros) }\end{array}$ & $\begin{array}{c}\text { DAP marginal } \\
\text { (ptas. de 1999) }\end{array}$ & $\begin{array}{c}\text { DAP marginal } \\
\text { (Euros) }\end{array}$ \\
\hline PICNIC & 724,36 & 4,35 & $-0,202$ & 0,0012 \\
COCHE & $-1436,166$ & $-8,63$ & $-662,036$ & $-3,98$ \\
SETAS & 960,454 & 5,77 & 226,850 & 1,36 \\
$\mathrm{CO}_{2}$ & 694,525 & 4,17 & 69,743 & 0,42 \\
EROSION & $-2,698$ & $-0,02$ & $-1,955$ & $-0,01$ \\
\hline
\end{tabular}

El diferente diseño experimental utilizado en la construcción de las alternativas no permite atribuir al distinto método la diferencia en las DAP marginales. A pesar de ello, la simulación mediante bootstraping de un diseño de efectos principales parece sugerir que la principal razón es el propio método de valoración y no el diseño utilizado. Por ello sería interesante una futura investigación en el análisis de la influencia de los métodos de preferencias declaradas en las respuestas de los individuos.

Al igual que en el trabajo de Mackenzie (1993), los valores que se estiman del método de ordenación y del método de elección simulado utilizando los mismos datos son similares. Sin embargo, esto no valida el supuesto de Mackenzie de que los métodos de preferencias declaradas por separado estiman a las mismas preferencias que si los datos se obtienen del mismo método. La diferencia estadísticamente significativa entre las medidas de bienestar derivadas de los datos pairwise originales de las derivadas de los datos pairwise simulados cuestionan este supuesto.

Por último, una evaluación más satisfactoria de los resultados tendría que tener en cuenta la influencia en los mismos del diferente número de observaciones entre los métodos de preferencias declaradas que se comparan, así como el supuesto de la independencia de las alternativas irrelevantes que hay detrás de los modelos econométricos utilizados.

\section{Bibliografía}


Adamowicz, W.L., Louviere, J.J., y Williams, M.(1994). Combining stated and revealed preference methods for valuing environmental amenities. Journal of Environmental Economics and Management, 26: 271-292.

Adamowicz, W.L., Boxall, P.C., Williams, M. y Louviere, J.J. (1998). Stated preference approaches for measuring passive use values: choice experiments and contingent valuation. American Journal of Agricultural Economics, 80: 65-75.

Beggs, S., Cardell, S. y Hausman, J.A. (1981). Assessing the potential demand for electric cars. Journal of Econometrics, 16(1): 1-19.

Ben-Akiva, M. y Lerman, S.R. (1985). Discrete choice analysis: theory and application to travel demand. MIT Press, Cambridge, Mass.

Boxall, P., Adamowicz, W.L., Williams, M., Swait, J. y Louviere, J.J. (1996). A comparison of stated preference approaches to the measurement of environmental values. Ecological Economics, 18: 243-253.

Chapman, R.G. y Staelin, R. (1982). Exploting rank ordered choice set data within the stocastic utility model. Journal of Marketing Research, 19: 288-301.

Departament de Medi Ambient (1996). Les emissions a l'atmosfera a Catalunya. 1996 Una aproximació quantitativa. Quaderns de medi ambient. Número 5. Generalitat de Catalunya, Barcelona.

Desvousges, W.H., Smith, V.K. y McGivney, M.P. (1983). A comparison of alternative approaches for estimating reaction and related benefits of water quality. EPA-230-0583-001. Office of Policy Analysis, U.S. Environmental Protection Agency. Washington, D.C.

Domencich, T. y McFadden, D. (1975). Urban travel demand: a behavioural approach. Amsterdam. North-Holland.

Efron, B. y Tibshirani, R.J. (1993). An introduction to the bootstrap. Chapman and Hall, New York.

Garrod, G.D. y Willis, K.G. (1997). The non-use benefits of enhancing forest biodiversity: A contingent ranking study. Ecological Economics, 21: 45- 61.

Garrod, G.D. y Willis, K.G. (1998). Using contingent ranking to estimate the loss of amenity value for inland waterways from public utility service structures. Environmental and Resource Economics, 12: 241-247.

Hahn, G.J. y Shapiro, S.S. (1966). A catalogue and computer programme for design and analysis of orthogonal symmetric and asymmetric fractional experiments. General Electric Research and Development Centre Report No 66-C-165, Schenectady, New York.

Hanley, N., MacMillan, D., Wright, R.E., Bullock, C., Simpson, I., Parsisson, D. y Crabtree, B. (1998a). Contingent valuation versus choice experiments: estimating the 
benefits of environmentally sensitive areas in Scotland. Journal of Agricultural Economics, 49(1): 1-15.

Hanley, N., Wright, R.E. y Adamowicz, W. (1998b). Using choice experiments to value the environment. Environmental and Resource Economics, 11(3-4): 413-428.

Krinsky, I. y Robb, L.A. (1986). On approximating the statistical properties of elasticities. The Review of Economics and Statistics, 68: 715-719.

Lareau, T.J. y Rae, D.A. (1989). Valuing WTP for diesel odor reductions: an application of contingent ranking technique. Southern Economic Journal, 55(3): 728742.

Louviere, J.J. y Woodworth, G.G. (1983). Design and analysis of simulated consumer choice or allocation experiments: an approach based on aggregate data. Journal of Marketing Research, 20: 350-367.

Luce, R.D. (1959). Individual choice behaviour: a theoretical analysis. John Wiley \& Sons, New York.

Mackenzie, J. (1993). A comparison of contingent preference models. American Journal of Agricultural Economics, 75: 593-603.

Magat, W.A., Viscusi, W.K. y Hurber, J. (1988). Paired comparison and contingent valuation approaches to morbidity risk valuation. Journal of Environmental Economics and Management, 15: 395-411.

McFadden, D. (1973). Conditional logit analysis of qualitative choice behaviour. In. P. Zarembka (Ed.), Frontiers in Econometrics, New York: Academic Press: 105-142.

Mitchell, R.C. y Carson, R.T. (1989). Using surveys to value public goods: the contingent valuation method. Resources for the Future, Washington, DC.

Morrison, M., Blamey, R.K., Bennett, J.W. y Louviere, J.J. (1996). A comparison of stated preference techniques for estimating environmental values. Research report, 1. School of Economics and Management, University College, University of New South Wales, Canberra.

Mourauto, S. y Foster, V. (1999). Elicitation format and part whole bias: do contingent valuation and contingent ranking give the same result? CSERGE working paper GEC 99-17.

Pearmin, D., Swanson, J., Kroes, E. y Bradley, M. (1991). Stated preference techniques. A guide to practique. Steer Davies Gleave y Hague Consulting Group, Londres.

Poe, G., Welsh, M. y Champ, P. (1997). Measuring the difference in mean willingness to pay when dichotomous choice contingent valuation responses are not independent. Journal of Environmental Economics and Management, 73: 255-267. 
Riera, P. (1995). Valoración de las externalidades de la emisión de gases contaminantes. Efectos en España y otros países Europeos. Instituto Universitario de Estudios Europeos, Universitat Autònoma de Barcelona, Barcelona.

Roe, B., Boyle, K. J. y Teisl M.F. (1996). Using conjoint analysis to derive estimates of compensating variation. Journal of Environmental Economics and Management, 31:145-159.

Rolfe, J., Bennett, J y Louviere, J.J. (2000). Choice modelling and its potential application to tropical rainforest preservation. Ecological Economics, 35: 289-302.

Swait, J., Louviere, J.J. (1993). The role of the scale parameter in the estimation and comparison of multinomial logit models. Journal of Marketing Research, 30: 305-14.

Veall, M.R.y Zimmermann, K.F. (1992). Pseudo-R ${ }^{2}$ in the ordinal probit model. Journal of Mathematical Sociology, 16: 333-342. 


\begin{tabular}{|c|c|c|}
\hline \multicolumn{3}{|c|}{ REFORESTACION 1} \\
\hline UTILIDAD DEL BOSQUE & & \\
\hline \multirow{3}{*}{$\begin{array}{l}\text { ACTIVIDADES } \\
\text { RECREATIVAS } \\
\text { PERMITIDAS }\end{array}$} & $\begin{array}{l}\text { - SE PUEDE CIRCULAR } \\
\text { CON VEHICULO POR LOS } \\
\text { CAMINOS } \\
\end{array}$ & $\begin{array}{ll}\text { - SE PUEDE CIRCULAR } \\
\text { CON VEHICULO POR } \\
\text { LOS CAMINOS }\end{array}$ \\
\hline & - PASEAR & - PICNIC \\
\hline & & - PASEAR \\
\hline $\begin{array}{l}\text { GAS } \mathrm{CO}_{2} \text { ELIMINADO AL } \\
\text { AÑO (Contaminación } \\
\text { producida por ... }\end{array}$ & $\begin{array}{l}\text { ciudad } \mathbf{4 0 0 . 0 0 0} \\
\text { habitantes }\end{array}$ & $\begin{array}{l}\text { ciudad } \mathbf{5 0 0 . 0 0 0} \\
\text { habitantes }\end{array}$ \\
\hline $\begin{array}{c}\text { LOS NUEVOS BOSQUES EN } \\
\ldots\end{array}$ & $\begin{array}{c}\text { tierras POCO } \\
\text { erosionadas } \\
\text { (improductivas en } 500 \text { años) }\end{array}$ & $\begin{array}{c}\text { tierras POCO } \\
\text { erosionadas } \\
\text { (improductivas en } \mathbf{5 0 0} \text { años) }\end{array}$ \\
\hline \multicolumn{3}{|l|}{ COSTE ECONÓMICO } \\
\hline CONTRIBUCIÓN ANUAL & 3.000 ptas/año & 4.000 ptas/año \\
\hline
\end{tabular}

Apéndice A. Ejemplo de uno de los conjuntos de elección presentados en el experimento de elección

\section{REFORESTACIÓN A}

\section{UTILDAD DEL BOSQUE}

\begin{tabular}{|c|c|}
\hline \multirow{4}{*}{$\begin{array}{l}\text { ACTIVIDADES } \\
\text { RECREATIVAS } \\
\text { PERMITIDAS }\end{array}$} & \begin{tabular}{|l|l|} 
& SE PUEDE CIRCULAR \\
CON VEHICULO POR LOS \\
CAMINOS
\end{tabular} \\
\hline & - BUSCAR SETAS \\
\hline & - PICNIC \\
\hline & 市 - PASEAR \\
\hline $\begin{array}{c}\text { GAS CO}_{2} \\
\text { ELIMINADO AL } \\
\text { AÑN (Contaminación } \\
\text { producida por ... }\end{array}$ & $\begin{array}{l}\text { ciudad } \mathbf{3 0 0 . 0 0 0} \\
\text { habitantes }\end{array}$ \\
\hline $\begin{array}{l}\text { LOS NUEVOS } \\
\text { BOSQUES EN ... }\end{array}$ & $\begin{array}{c}\text { tierras MUY POCO } \\
\text { erosionadas } \\
\text { (improductivas en } 700 \text { años) }\end{array}$ \\
\hline \multicolumn{2}{|l|}{ EOSTE ECONÓMIEO } \\
\hline $\begin{array}{l}\text { CONTRIBUCIÓN } \\
\text { ANUAL }\end{array}$ & 1.500 ptas /año \\
\hline
\end{tabular}

